\title{
Effect of optimized structure and electronic properties of some benzimidazole derivatives on corrosion inhibition of mild steel in hydrochloric acid medium: Electrochemical and theoretical studies
}

\author{
ALOKDUT DUTTA, SUJIT SANKAR PANJA, M M NANDI and DIPANKAR SUKUL* \\ Department of Chemistry, National Institute of Technology Durgapur, Durgapur 713 209, India \\ e-mail: dipankar.sukul@gmail.com
}

MS received 30 July 2014; revised 26 November 2014; accepted 6 January 2015

\begin{abstract}
The corrosion inhibitive action of a few benzimidazole derivatives namely 2-(benzamido) ethylbenzimidazole (BAEBI), 2-( $\beta$-benzenesulphonamido) ethylbenzimidazole (BSAEBI), 2-(benzamido) methylbenzimidazole (BAMBI) and 2-( $\beta$-benzenesulphonamido) methylbenzimidazole (BSAMBI), towards mild steel in hydrochloric acid has been studied using potentiodynamic polarization and electrochemical impedance spectroscopic (EIS) methods. The results show that these compounds get adsorbed on the mild steel surface following Temkin adsorption isotherm, and act as mixed-type inhibitors. The inhibition efficiencies are found to follow the order, BAEBI $>$ BSAEBI $>$ BAMBI > BSAMBI. This observation is explained in terms of chain length, relative effects of amido and sulphonamido groups, possible structural factors, spatial orientations, energy gap between the frontier molecular orbitals, different intrinsic molecular parameters, like, global hardness and softness, and number of electrons transferred.
\end{abstract}

Keywords. Adsorption; corrosion inhibition; electrochemical techniques; quantum mechanical calculations.

\section{Introduction}

Organic inhibitors including aromatic heterocycles containing sulfur, oxygen and nitrogen are effectively used to minimize the aggressiveness of acids towards corrosion of metals. Replacing the surface adsorbed water molecules, these inhibitors get adsorbed on the metal surface, and thereby diminish the rate of either cathodic reduction reaction, or anodic metal dissolution reaction, or both. Various approaches have been made to correlate the extent of adsorption, and subsequent inhibitory action with molecular parameters of the inhibitors. These include the presence of lone pair of electrons on heterocyclic atoms, chain length (i.e., molecular volume), presence of unsaturated bond, aromatic substituent group, planarity of heterocycles, steric factor, HOMO-LUMO energy gap of the molecules, and other molecular parameters. ${ }^{1-12}$

Several classes of $N$-heterocyclic compounds including benzimidazole (BI) and its derivatives have drawn considerable attention as acid corrosion inhibitors, mainly due to their significant corrosion inhibition efficiency, good chelating ability, relatively higher solubility in acid media and also their environment-friendly properties. ${ }^{13-17}$

\footnotetext{
*For correspondence
}

In the present study, we have used four different benzenesulphonamido and benzamido derivatives of BI (table 1) to investigate their corrosion inhibitory properties for mild steel in $1 \mathrm{M} \mathrm{HCl}$. Sulphonamides and benzamides are also known for their antimicrobial properties. ${ }^{18-20}$ Our main endeavour is to correlate the corrosion inhibition potential with the structure and molecular parameters of BI derivatives where benzenesulphonamido and benzamido groups are separated from the BI moiety by one and two methylene groups. Corrosion inhibition efficiency of these compounds is determined using different electrochemical techniques, like potentiodynamic polarization and electrochemical AC impedance. Structure effect of the inhibitors towards corrosion inhibition of mild steel is evaluated by quantum chemical calculations and comparing various molecular parameters, such as energy optimized geometry, HOMO and LUMO energy levels and other molecular parameters, which include global hardness and softness, and number of electron transferred.

\section{Experimental}

\subsection{Synthesis of inhibitors}

All the inhibitors were prepared in simple two steps reactions as per reported procedure. ${ }^{21-23}$ In short, for 
Table 1. Structures and characteristics of benzimidazole derivatives.

$\begin{array}{ll}\text { Molecules } & \begin{array}{c}\text { Characteristic } \\ \text { IR peaks }\left(\mathrm{cm}^{-1}\right)\end{array} \\ \text { 2-(benzamido) } \\ \text { methylbenzimidazole } \\ \text { ethylbenzimidazole } \\ \text { ethylbenzimidazole }\end{array}$

preparing BSAEBI and BSAMBI, benzenesulfonyl chloride was added drop wise to the vigorously stirred aqueous solution of glycine and alanine, respectively (1:1 mole ratio), maintaining $\mathrm{pH}$ of the solution at 9 . After complete addition, the mixture was stirred further for $15 \mathrm{~min}$ followed by the addition of $4.5 \mathrm{M} \mathrm{H}_{2} \mathrm{SO}_{4}$ to adjust $\mathrm{pH}$ at 2 . The precipitate was filtered out, washed and recrystallized from aqueous ethanol mixed solvent. It was then refluxed with o-phenylenediamine (1:1 mole ratio) for $3 \mathrm{~h}$ in $2 \mathrm{M} \mathrm{H}_{2} \mathrm{SO}_{4}$. Mixture was cooled at room temperature and $\mathrm{pH}$ was adjusted to 9 by adding $10 \% \mathrm{NaOH}$ and $7 \mathrm{M} \mathrm{NH}_{3}$ (aq). The final compound which precipitated, was filtered, washed and recrystallized from aqueous ethanol mixed solvent. For preparing BAEBI and BAMBI, same procedure was adopted with the use of benzoyl chloride, and the final $\mathrm{pH}$ was adjusted to 6-7. These compounds were characterized by FT-IR (Thermo-Nicolet iS10 IR spectrometer in the range $4000-400 \mathrm{~cm}^{-1}$ ) and melting point measurement (tabulated in table 1)..$^{21,22}$

\subsection{Specimens and solution}

Cylindrical specimens were prepared by cutting commercially available mild steel rod (wt\% composition: $0.22 \mathrm{C}, 0.31 \mathrm{Si}, 0.60 \mathrm{Mn}, 0.04 \mathrm{P}, 0.06 \mathrm{~S}$ and the remainder iron). Prior to each experiment, the surfaces were pre-treated by grinding on a belt grinding polishing machine followed by metallurgical grade emery papers of 400-1600 grit. Then the samples were degreased with ethanol and rinsed with bi-distilled water. $\mathrm{HCl}$
(1 M) solution was prepared using 35\% $\mathrm{HCl}$ (GR grade, Merck India).

\subsection{Electrochemical studies}

Potentiodynamic polarization and electrochemical impedance measurement were done by a conventional three-electrode system (model: Gill AC, ACM Instruments, UK) consist of mild steel as a working electrode (WE) with exposed area of $0.25 \mathrm{~cm}^{2}$, large area platinum foil as counter electrode and saturated calomel electrode (SCE) as the reference. Before measurements, the WE was kept in contact with test solution $(200 \mathrm{~mL}$ ) for $45 \mathrm{~min}$ to achieve a steady state (by observing variation of potential and current with time). The potential sweep rate for potentiodynamic polarization curves was $0.5 \mathrm{mV} / \mathrm{sec}$. Corrosion current density $\left(i_{\text {corr }}\right)$ was determined from the intercept of the extrapolated cathodic and anodic Tafel lines at the corrosion potential $\left(E_{\text {corr }}\right)$. Electrochemical impedance measurements were performed in the frequency range $10 \mathrm{mHz}$ to $100 \mathrm{kHz}$ with a.c. amplitude of $\pm 10 \mathrm{mV}$ (r.m.s.) at the open circuit potential (OCP). All the experiments were done at around the room temperature of $30^{\circ} \mathrm{C}$.

\subsection{Theoretical calculations}

All the quantum chemical ground electronic state calculations in gas phase were performed using Gaussian09 program. ${ }^{24}$ Geometry optimization and determination 
of energy of frontier molecular orbitals for all the molecules have been carried out considering density functional theory (DFT) at the B3LPY level through 6$31++\mathrm{g}(\mathrm{d}, \mathrm{p})$ basis set. The frequency calculations are done with the same basis set. All the frequencies are found to be positive, which reflects that the ground state optimized structures correspond to global minima. In addition to the energies of the highest occupied molecular orbital $\left(E_{\text {Номо }}\right)$, and lowest unoccupied molecular orbital $\left(E_{\mathrm{LUMO}}\right)$, other molecular parameters of the inhibitors, like electronegativity $(\chi)$, global hardness $(\eta)$ and softness $(\sigma)$, electron affinity $(A)$, ionization potential $(I)$, and number of electrons transferred $(\Delta N)$ have been calculated.

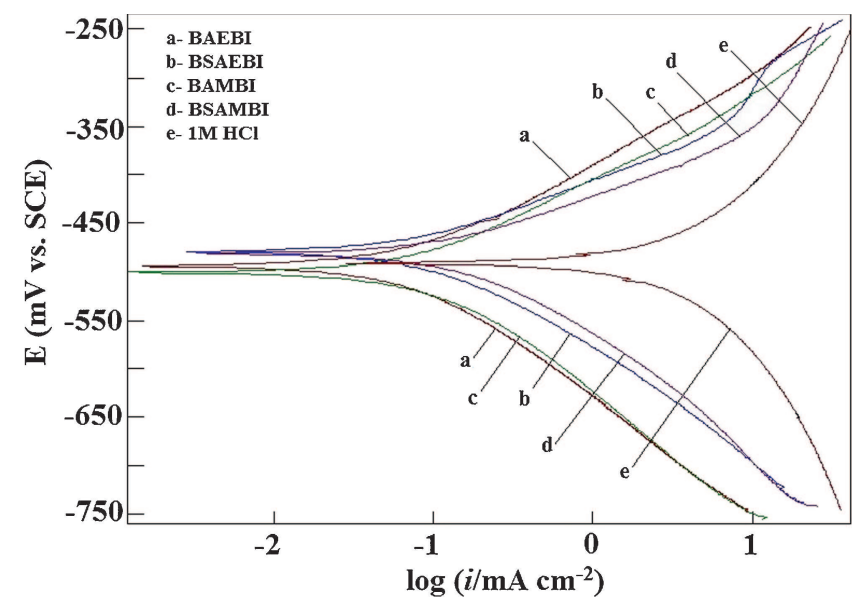

Figure 1. Potentiodynamic polarization curves for mild steel in $1 \mathrm{M} \mathrm{HCl}$ in the presence of $\mathrm{BI}$ derivatives having 1 $\mathrm{mM}$ concentration.

\section{Results and Discussion}

\subsection{Polarization studies}

Potentiodynamic polarization curves for mild steel in $1 \mathrm{M} \mathrm{HCl}$ and in presence of the inhibitors used having $1 \mathrm{mM}$ concentration are shown in figure $1(\mathrm{a}-\mathrm{e})$. Variations of cathodic and anodic currents with potential for all the four inhibitors with $0.1 \mathrm{mM}$ and $1 \mathrm{mM}$ concentrations are shown in figure $\mathrm{S} 1(\mathrm{a}-\mathrm{d})$. Values of the electrochemical corrosion parameters, such as corrosion potential $\left(E_{\text {corr }}\right)$, cathodic Tafel slope $\left(b_{c}\right)$, anodic Tafel slope $\left(b_{\mathrm{a}}\right)$, and corrosion current density $\left(i_{\text {corr }}\right)$ for all the inhibitors having concentration range of 0.1 to 1 $\mathrm{mM}$ are given in table 2 . The percentage inhibition efficiency $\eta_{\mathrm{P}}(\%)$ is calculated from the following equation:

$$
\eta_{\mathrm{P}}(\%)=\frac{i_{\text {corr }}-i_{\text {corr(inh) }}}{i_{\text {corr }}} \times 100
$$

where, $i_{\text {corr }}$ and $i_{\text {corr(inh) }}$ are the values of corrosion current density of uninhibited and inhibited specimens, respectively. A gradual increment in corrosion inhibition efficiency is seen with increase in inhibitor concentration. Figure S1 (a-d) clearly shows that with increase in inhibitor concentration, both the cathodic and anodic current decreases. Furthermore, no regular variation of $E_{\text {corr }}$ values with concentration of the inhibitors can be observed (table 2). These observations suggest that the compounds behave as mixed type inhibitors which reduce both the rate of cathodic and anodic reactions by blocking the respective reaction sites on the metal surface.

Table 2. Data from potentiodynamic polarization studies for mild steel in $1 \mathrm{M} \mathrm{HCl}$ in various inhibitor systems.

\begin{tabular}{|c|c|c|c|c|c|c|}
\hline System & Conc. (mM) & $-E_{\text {corr }}(\mathrm{mV} / \mathrm{SCE})$ & $i_{\text {corr }}\left(\mu \mathrm{A} \mathrm{cm}^{-2}\right)$ & $b_{\mathrm{a}}\left(\mathrm{mVdec}^{-1}\right)$ & $-b_{\mathrm{c}}\left(\mathrm{mVdec} \mathrm{de}^{-1}\right)$ & $\eta_{\mathrm{p}}(\%)$ \\
\hline BLANK & & 491 & 1200 & 65 & 77 & \\
\hline \multirow[t]{4}{*}{ BAEBI } & 0.10 & 489 & 525 & 77 & 107 & 56 \\
\hline & 0.75 & 486 & 148 & 80 & 104 & 88 \\
\hline & 0.90 & 500 & 123 & 91 & 129 & 90 \\
\hline & 1.00 & 494 & 66 & 80 & 119 & 95 \\
\hline \multirow[t]{4}{*}{ BSAEBI } & 0.10 & 495 & 685 & 81 & 106 & 43 \\
\hline & 0.75 & 471 & 231 & 75 & 119 & 81 \\
\hline & 0.90 & 483 & 156 & 85 & 112 & 87 \\
\hline & 1.00 & 478 & 102 & 71 & 105 & 92 \\
\hline \multirow[t]{4}{*}{ BAMBI } & 0.10 & 497 & 787 & 70 & 100 & 34 \\
\hline & 0.75 & 471 & 273 & 86 & 112 & 77 \\
\hline & 0.90 & 486 & 179 & 82 & 116 & 85 \\
\hline & 1.00 & 501 & 110 & 92 & 136 & 90 \\
\hline \multirow[t]{4}{*}{ BSAMBI } & 0.10 & 496 & 835 & 85 & 109 & 30 \\
\hline & 0.75 & 476 & 362 & 82 & 105 & 70 \\
\hline & 0.90 & 470 & 240 & 90 & 124 & 80 \\
\hline & 1.00 & 481 & 207 & 77 & 121 & 83 \\
\hline
\end{tabular}


It is observed that when benzenesulphonamido or benzamido groups are attached to the BI moiety through two methylene groups, inhibition efficiencies are higher than the corresponding compounds where one methylene group acts as a separator. This is in agreement with the general observation that inhibition efficiency increases with increase in chain length (i.e., molecular volume). ${ }^{5}$ Between benzenesulphonamido and benzamido substituted compounds, latter compounds show higher corrosion inhibition efficiency.

\subsection{Electrochemical impedance measurements}

The corrosion behaviour of mild steel in $\mathrm{HCl}$ in presence of inhibitors was investigated by EIS. Corresponding Nyquist plots for different inhibitor systems in $1 \mathrm{mM}$ concentration are given in figure 2. Bode plots are shown in figure S2 (a-d). From Bode magnitude and phase plots, it is observed that the metal-electrolyte interface in the present study is characterized by a single time constant system. Comparing the plots for all the inhibitor systems having concentration in the range of $0.1 \mathrm{mM}$ to $1 \mathrm{mM}$, it is seen that diameter of the capacitive loop increases with increase in concentration of all the inhibitors suggesting the formation of protective surface film. All the spectra have depressed semi-circle with centre under the real axis, which may be attributed to the roughness and inhomogeneities of solid surface. To analyse those, a model consisting of parallel combination of charge transfer resistance-constant phase element, which is in series with solution resistance, $R_{\mathrm{s}}\left[R_{\mathrm{ct}}-\right.$ $C P E]$ (figure S3), is used in the present study. ${ }^{25-27}$ The impedance of $C P E$ is given by

$$
\mathrm{Z}_{\mathrm{CPE}}=Q^{-1}(i \omega)^{-n}
$$

where, $Q$ is a proportionality coefficient, $\omega$ is the angular frequency, $\mathrm{n}$ is a measure of surface irregularity. $Q$ is

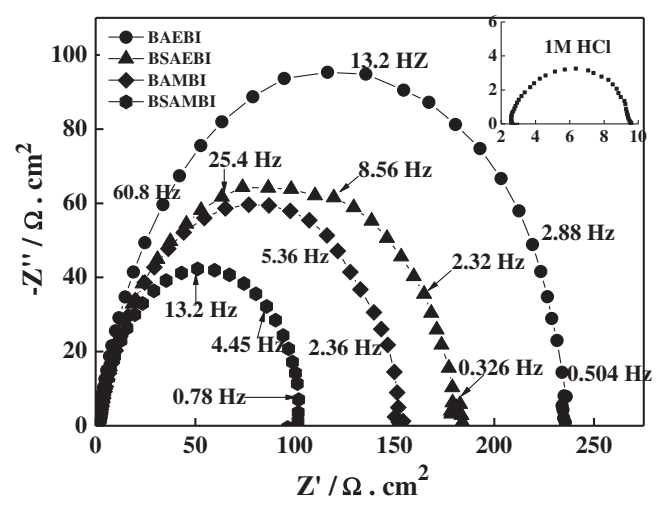

Figure 2. Nyquist plots for mild steel in $1 \mathrm{M} \mathrm{HCl}$ in the presence of BI derivatives $(1 \mathrm{mM})$. an indicative parameter proportional to the capacitance of the double layer formed at the metal surface for $0>$ $\mathrm{n}>1$. For whole numbers of $\mathrm{n}=1,0,-1, C P E$ is reduced to the classical lumped elements capacitor $(C)$, resistance $(R)$, and inductance $(L)$, respectively. The fitted parameters using the above model are presented in table 3 .

It is seen in table 3 that for all the inhibitors, the value of $R_{\mathrm{ct}}$ gradually increases with concentration, while, the value of $Q$ decreases. This confirms the adsorption of inhibitors on the metal surface which provide a corrosion inhibitive layer.

The percentage inhibition efficiencies $\eta_{Z}(\%)$ in terms of $R_{\mathrm{ct}}$ are calculated using eq. 3 .

$$
\eta_{\mathrm{Z}}(\%)=\frac{R_{\mathrm{ct}}-R_{\mathrm{ct}}^{0}}{R_{\mathrm{ct}}} \times 100
$$

where, $R_{\mathrm{ct}}$ and $R_{\mathrm{ct}}^{0}$ are the values of charge transfer resistance observed in the presence and absence of inhibitor, respectively. Following $R_{\mathrm{ct}}$ values, $\eta_{\mathrm{Z}}(\%)$ are seen to follow the order BAEBI $>$ BSAEBI $>$ BAMBI $>$ BSAMBI, i.e. the same trend as observed in the polarisation measurements.

\subsection{Adsorption isotherm}

To demonstrate the adsorption characteristics of the BI derivatives on mild steel surface, and to determine the free energy of adsorption, we have assessed several types of adsorption isotherms. Temkin adsorption isotherm, which is based on the consideration of

Table 3. Impedance parameters for the corrosion of mild steel in $1 \mathrm{M} \mathrm{HCl}$ in various inhibitor systems.

\begin{tabular}{llrccc}
\hline System & $\begin{array}{l}\text { Conc. } \\
(\mathrm{mM})\end{array}$ & $\begin{array}{c}R_{\mathrm{ct}} \\
\left(\Omega \mathrm{cm}^{2}\right)\end{array}$ & $\begin{array}{c}Q\left(\mu \Omega^{-1} \mathrm{~s}^{\mathrm{n}}\right. \\
\left.\mathrm{cm}^{-2}\right)\end{array}$ & $n$ & $\eta_{\mathrm{z}}(\%)$ \\
\hline BLANK & & 6.3 & 593 & 0.85 & \\
BAEBI & 0.1 & 23 & 457 & 0.84 & 72 \\
& 0.75 & 143 & 130 & 0.83 & 95 \\
& 0.9 & 164 & 122 & 0.83 & 96 \\
& 1.0 & 237 & 119 & 0.82 & 97 \\
BSAEBI & 0.1 & 20 & 816 & 0.75 & 67 \\
& 0.75 & 80 & 405 & 0.77 & 91 \\
& 0.9 & 128 & 282 & 0.80 & 94 \\
BAMBI & 1.0 & 185 & 172 & 0.75 & 96 \\
& 0.1 & 15 & 885 & 0.75 & 56 \\
& 0.75 & 52 & 751 & 0.70 & 88 \\
& 0.9 & 107 & 428 & 0.72 & 93 \\
BSAMBI & 0.1 & 149 & 114 & 0.85 & 95 \\
& 0.75 & 11 & 798 & 0.78 & 40 \\
& 0.9 & 66 & 725 & 0.72 & 85 \\
& 1.0 & 104 & 358 & 0.74 & 90 \\
& & & 304 & 0.81 & 93 \\
\hline
\end{tabular}




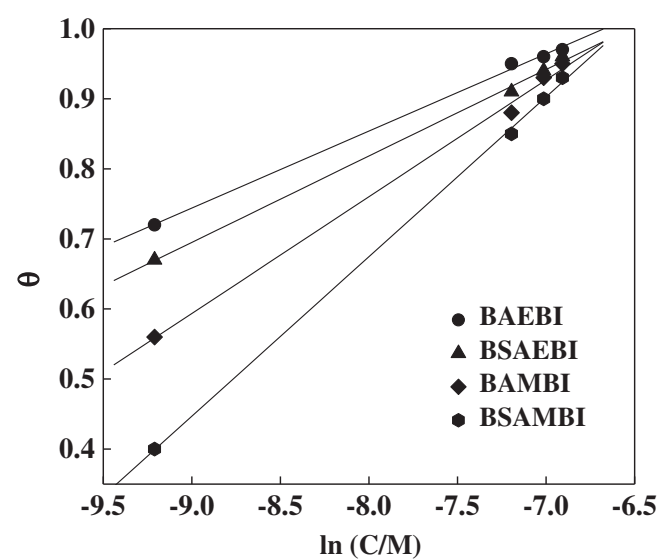

Figure 3. Temkin adsorption plots for mild steel in $1 \mathrm{M}$ $\mathrm{HCl}$ in presence of benzimidazole derivatives.

the heterogeneity of the surface, is found to be best suited for fitting of the experimental obtained results (figure 3, table 4) as evident from the minimum value of correlation coefficient, $r^{2}=0.995$. According to this model, degree of surface coverage $\theta\left(\theta=\frac{\eta_{z}(\%)}{100}\right)$ is related to the concentration of the inhibitor $(\mathrm{C})$ by the eq. $4: 28,29$

$$
\exp (f \theta)=K_{\text {ads }} \mathrm{C}
$$

where, $K_{\text {ads }}$ is the constant of adsorption and $f$ is the interaction parameter. Plotting $\theta$ versus $\ln \mathrm{C}$ and from its slope and intercept, the values of $f$ and $K_{\text {ads }}$ have been obtained (figure 3, table 4). The interaction parameter, $f$ is found to be positive and greater than 1 for all the inhibitors, which signifies the existence of molecular interaction in the adsorbed layer as well as energetic heterogeneity of the surface..$^{28,29}$

From the values of the adsorption constant, $K_{\text {ads }}$, the standard free energy of adsorption, $\Delta G_{\text {ads }}^{0}$ for all the inhibitors are determined using the following equation:

$$
\Delta G_{\mathrm{ads}}^{0}=-R T \ln \left(55.55 K_{\mathrm{ads}}\right)
$$

where, 55.55 is the molar concentration of water. $\Delta G_{\text {ads }}^{0}$ at $303 \mathrm{~K}$ for all the four inhibitors are shown in table 4 . Negative values of $\Delta G_{\mathrm{ads}}^{0}$ for all the inhibitors suggest

Table 4. Adsorption parameters for benzimidazole derivatives on mild steel in $1 \mathrm{M} \mathrm{HCl}$.

\begin{tabular}{lcccc}
\hline Sample & $\mathrm{r}^{2}$ & $f$ & $K_{\text {ads }}\left(\mathrm{dm}^{3} \mathrm{~mol}^{-1}\right)$ & $-\Delta G_{\text {ads }}^{0}\left(\mathrm{~kJ} \mathrm{~mol}^{-1}\right)$ \\
\hline BAEBI & 0.997 & 9.09 & $7.01 \times 10^{6}$ & 49.8 \\
BSAEBI & 0.997 & 8.10 & $2.26 \times 10^{6}$ & 46.9 \\
BAMBI & 0.995 & 5.99 & $2.85 \times 10^{5}$ & 41.7 \\
BSAMBI & 0.999 & 4.39 & $5.76 \times 10^{4}$ & 37.7 \\
\hline
\end{tabular}

spontaneous adsorption of the inhibitor molecules on mild steel surface in acid medium. ${ }^{30-33}$ BAEBI is seen to have the maximum potentiality of adsorption, while BSAMBI having the least.

It is proposed in literature that BI derivatives may interact with metal surface in acid medium in two ways. ${ }^{15}$ Either the neutral form of the derivatives may directly interact with metal surface (i.e., electron transfer from the inhibitors to the positively charges metal surface), or protonated form of the derivatives can be adsorbed on the metal surface through pre-adsorbed $\mathrm{Cl}^{-}$ ions. But, it is well-known that for $3 \mathrm{~d}$ metal ions (particularly for $\mathrm{Fe}^{2+}, \mathrm{Cu}^{2+}, \mathrm{Ni}^{2+}, \mathrm{Co}^{2+}$ ), the formation constant of chloro-aqua complex is very low corresponding to hexa-aqua ions. ${ }^{34} \mathrm{In} 1 \mathrm{M} \mathrm{HCl}$, the predominant chloro complex for $\mathrm{Fe}^{3+}$ is $\left[\mathrm{FeCl}\left(\mathrm{H}_{2} \mathrm{O}\right)_{5}\right]^{2+}$. Anionic chloro complex of $\mathrm{Fe}^{3+}$ (i.e. $\mathrm{FeCl}_{4}^{-}$) is only stable in concentrated $\mathrm{HCl}^{34}$ On the other hand, $\mathrm{BI}$ ligands in $1 \mathrm{M} \mathrm{HCl}$ mostly present in protonated form $\left(\mathrm{pK}_{\mathrm{a}}\right.$ value of the protonated BI and its derivatives being within $4-5) .{ }^{35,36}$ However, in presence of metal ions, BI ligands should prefer to bind with more positively charged metal ions over singly positively charged protons. This is supported from the observation that formation constant for $\mathrm{BI}$ derivatives-Metal (II) complexes is much higher than that for protonated BI derivatives. ${ }^{35,36}$ Hence, it may be presumed that during adsorption, $\mathrm{H}^{+}$from the protonated inhibitor and $\mathrm{Cl}^{-}$from the metal surface will be displaced as $\mathrm{HCl}$ and complexes involving the neutral inhibitor molecules and metal ions will be formed at the metal surface.

\subsection{Quantum chemical study and mechanism of inhibition action}

In general, electron donating ability of a molecule increases with increase in $E_{\mathrm{HOMO}}$, whereas, a lower value of $E_{\text {Lumo }}$ indicates the molecule to be more susceptible towards accepting electrons. ${ }^{37,38}$ Thus, adsorption of an inhibitor and its corrosion inhibition efficiency is characterized by increased $E_{\text {HOMO }}$, decreased $E_{\mathrm{LUMO}}$ and a low value of the energy gap $\Delta E=$ $E_{\mathrm{LUMO}}-E_{\mathrm{HOMO}}$. Electron distribution in the optimized HOMO and LUMO energy levels of the inhibitors are shown in figure 4 and the corresponding energy values are tabulated in table 5 . It is seen that $E_{\text {Номо values }}$ increase linearly with increase in their corresponding IE values (with a correlation factor of 0.987 ) (figure 5). For $E_{\text {LUMO }}$, such a nice linear variation is absent. Though $\Delta E$ decreases with increase in inhibition efficiency, it lacks sufficient linearity (correlation factor of 0.925) (figure 5). All these observation indicate that adsorption of the inhibitors studied is mostly governed by the 


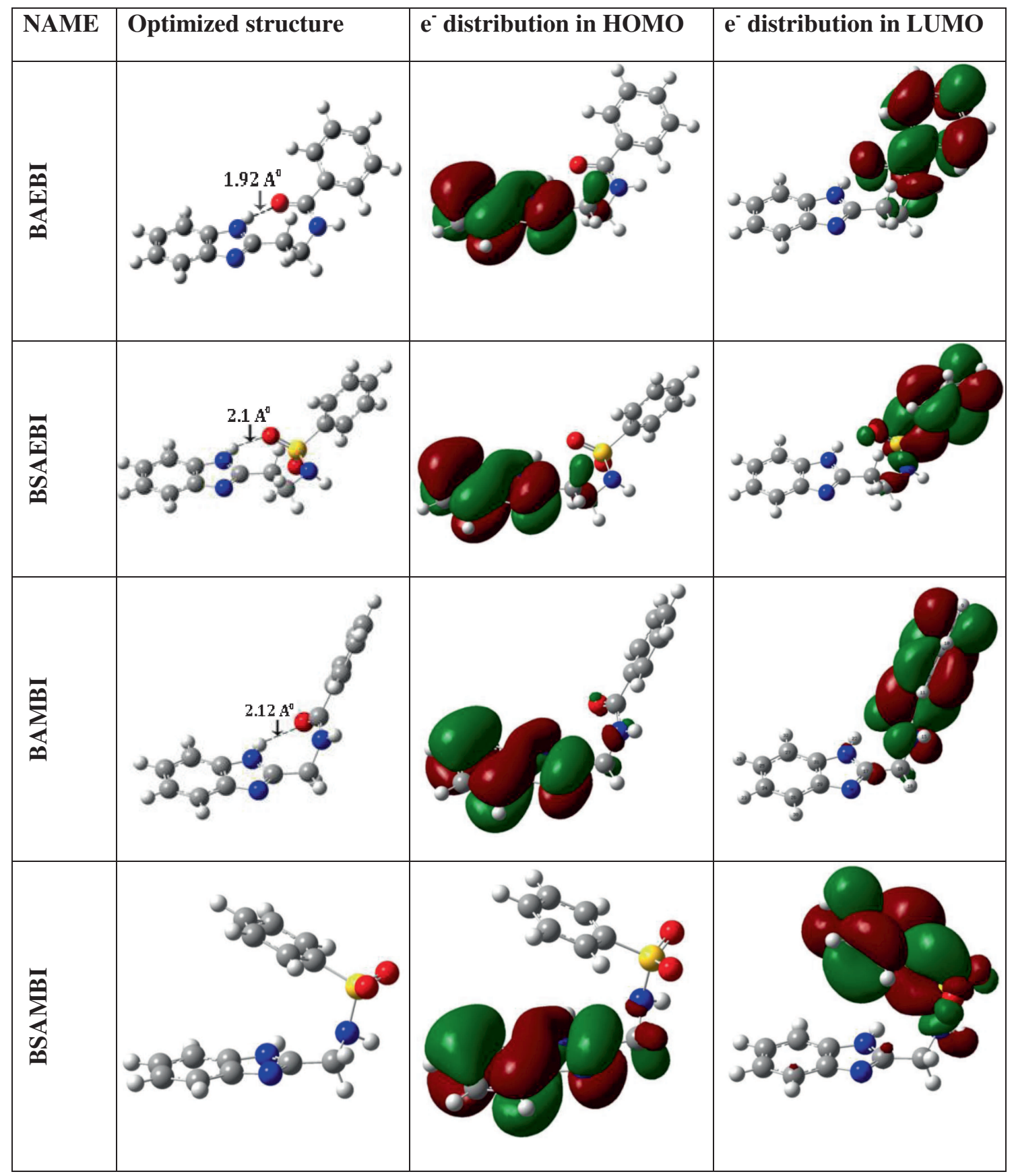

Figure 4. Optimized molecular structures of benzimidazole derivatives with HOMO and LUMO orbitals.

electron transfer from the HOMO of the inhibitors to the vacant metal d orbitals. Extent of any possible back donation of metal $4 \mathrm{~s}$ electrons to the LUMO of the inhibitors is not the same for all the inhibitor, which is reflected in the observed variation of inhibition efficiency with $E_{\mathrm{LUMO}}$ and $\Delta E$ values. Above findings may

Table 5. The calculated quantum chemical parameters for benzimidazole derivatives with DFT method.

\begin{tabular}{lccccccccc}
\hline System & $E_{\text {Номо }}(\mathrm{eV})$ & $E_{\text {LUMO }}(\mathrm{eV})$ & $\Delta E(\mathrm{eV})$ & $I(\mathrm{eV})$ & $A(\mathrm{eV})$ & $\chi(\mathrm{eV})$ & $\eta(\mathrm{eV})$ & $\sigma\left(\mathrm{eV}^{-1}\right)$ & $\Delta N$ \\
\hline BAEBI & -5.90 & -1.78 & 4.12 & 5.90 & 1.78 & 3.84 & 2.06 & 0.485 & 0.24 \\
BSAEBI & -6.00 & -1.83 & 4.17 & 6.00 & 1.83 & 3.915 & 2.085 & 0.48 & 0.22 \\
BAMBI & -6.18 & -1.68 & 4.50 & 6.18 & 1.68 & 3.93 & 2.25 & 0.44 & 0.20 \\
BSAMBI & -6.41 & -1.64 & 4.77 & 6.41 & 1.64 & 4.02 & 2.38 & 0.42 & 0.17 \\
\hline
\end{tabular}




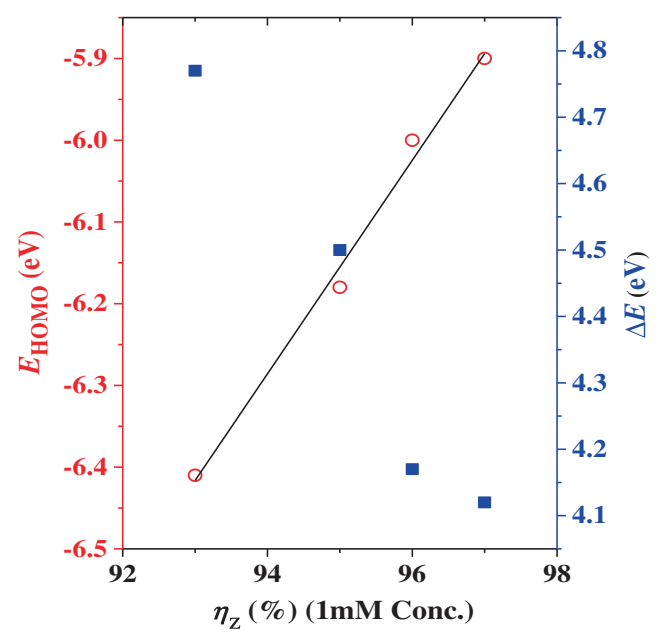

Figure 5. Correlation of (a) $E_{\text {HOMO }}\left(\mathrm{O}_{-}\right)$and (b) energy gap $\left(\Delta E=E_{\mathrm{LUMO}}-E_{\mathrm{HOMO}}\right)(\square)$ with percent inhibition efficiency of benzimidazole derivatives $(1 \mathrm{mM})$.

be interpreted in terms of the optimized geometry of the inhibitor molecules. It is seen that HOMO energy level of all the molecules is mostly distributed over planar BI group, while LUMO energy level is on the benzenesulphonamido or benzamido substituents. For BAEBI, BSAEBI and BAMBI, strong $\mathrm{H}$ bond is seen to exist (1.9 $\AA$ to $2.12 \AA$ ) between the $\mathrm{H}$ atom attached to $\mathrm{N}$ atom of $\mathrm{BI}$ moiety and $\mathrm{O}$ atom of carbonyl or sulfonyl group. This provides sufficient rigidity to the molecules that BI plane and the aromatic plane of benzenesulphonamido or benzamido groups are oriented in such a fashion which is not conducive for simultaneous two ways bonding, i.e., electron donation from HOMO of inhibitors to the vacant iron $3 \mathrm{~d}$ orbitals, and the back electron transfer from metal $4 \mathrm{~s}$ orbital to LUMO energy levels of the inhibitors, with comparable extent. From the optimized geometry of BSAMBI, which exhibits least corrosion inhibition efficiency, it is observed that benzene of benzenesulphonamido moiety is almost poised over the BI group. Such spatial orientation is ideal for non-bonded aromatic $\pi-\pi$ interaction which reduces the $\pi$-electron density of BI moiety and subsequent reduction in the extent of adsorption. ${ }^{39}$

Following Koopmans' theorem, different intrinsic molecular quantities like electronegativity $(\chi$, a measure of the power of a group of atoms to attract electrons towards itself), global hardness ( $\eta$, a parameter related to the resistance of an atom to a charge transfer) and global softness ( $\sigma$, which shows the reactivity of the inhibitor molecules in terms of charge transfer), and number of electrons transferred $(\Delta N)$, which are supposed to influence the overall reaction between two interacting systems, have been calculated (table 5). ${ }^{40-42}$
These quantities are related to electron affinity (A) and ionization potential (I) as follows:

$$
\begin{gathered}
\chi=(I+A) / 2 \\
\eta=(I-A) / 2 \\
\sigma=1 / \eta=2 /(I-A) \\
I=-E_{\mathrm{HOMO}} \\
A=-E_{\mathrm{LUMO}}
\end{gathered}
$$

From these values, the number of electrons transferred $(\Delta N)$, from inhibitor molecules having lower electronegativity to the more electronegative iron surface until the chemical potential becomes equalized, are computed using the equation (table 5):

$$
\Delta N=\frac{\left(\chi_{\mathrm{Fe}}-\chi_{\mathrm{inh}}\right)}{2\left(\eta_{\mathrm{Fe}}+\eta_{\mathrm{inh}}\right)}
$$

where, $\chi_{\mathrm{Fe}}$ is taken as $4.82 \mathrm{eV} / \mathrm{mol}$, equivalent to the work function for Fe (110) surface and $\eta_{\mathrm{Fe}}$ is 0 $\mathrm{eV} / \mathrm{mol}^{43}$ Electronegativity values of all the inhibitor molecules are found to be lower than that of iron, suggesting electron flow from the HOMO of the inhibitors towards the vacant $3 \mathrm{~d}$ orbitals of $\mathrm{Fe}$ to be more significant than that from the filled $4 \mathrm{~s}$ orbital of Fe to LUMO of the inhibitors. Among benzenesulphonamido and benzamido substituted compounds, latter compounds show lower $\chi$, which may be attributed towards higher electronegativity of $\mathrm{S}$ atom over $\mathrm{C}$ atom, as well as presence of more number of highly electronegative $\mathrm{O}$ atoms in sulphonamido group. As a result, electron flow from benzamido substituted compounds is potentially more favourable than the others, resulting in higher inhibition efficiency. Also, it is seen from the table 5 that inhibition efficiency increases when global hardness decreases and global softness increases. Thus, with decrease in energy gap between the frontier molecular orbitals of the inhibitors, reactivity of molecules towards charge transfer with metal atoms increases. This is manifested in increasing number of electron transferred $(\Delta N)$. According to Lukovits's study, ${ }^{44}$ if the value of $\Delta N<3.6$, the inhibition efficiency is increased with increasing electron donating ability of inhibitor at the metal surface. For BSAMBI, which has least inhibition efficiency, $\Delta N$ is seen to be 0.17 , whereas for BAEBI having highest inhibition efficiency, $\Delta N$ is 0.24 . 


\section{Conclusions}

It is observed that different structural aspects like spatial orientation, chain length, presence of hetero atoms and energies of frontier molecular orbitals provide profound influence on the adsorption and subsequent corrosion inhibition by the BI derivatives used in this present study. Inhibitors having higher chain length (i.e., molecular volume) provide higher inhibition efficiency. Between benzenesulphonamido and benzamido substituted compounds, latter compounds show higher corrosion inhibition efficiency, which may be attributed to higher electronegativity of $\mathrm{S}$ atom over $\mathrm{C}$ atom, as well as presence of higher number of highly electronegative $\mathrm{O}$ atoms in sulphonamido group. Inhibition efficiency is also found to depend on the spatial orientation of the substituents relative to the BI moiety. All the molecules are found to act as mixed type inhibitors. From adsorption isotherm studies (Temkin adsorption isotherm), it may be argued that inhibitors adsorb spontaneously on the mild steel surface. Quantum mechanical calculations suggest that during chemical adsorption, electrons are transferred from HOMO energy level of the molecules to the vacant $d$ orbitals of iron. As the electron density in the HOMO level is mostly distributed over the planar BI moiety, it is proposed that inhibitors are adsorbed parallel to the metal surface through the BI moiety. A linear correlation is found to exist between the inhibition efficiencies and $E_{\mathrm{HO} о}$ of the inhibitors. Other intrinsic molecular properties based on the energies of frontier molecular orbitals also explain quite satisfactorily the observed anti-corrosive characteristics of the inhibitors.

\section{Supplementary Information}

Supplementary material includes potentiodynamic polarizarions curves for all the inhibitors with $1 \mathrm{M}$ and $0.1 \mathrm{M}$ concentrations (figures $\mathrm{S} 1 \mathrm{a}-\mathrm{d}$ ), Bode plots for all the inhibitors with $1 \mathrm{M}$ concentration (figures $\mathrm{S} 2 \mathrm{a}-\mathrm{d}$ ), and diagram of the equivalent circuit used to fit the EIS data (figure S3) and the SEM images of the corroded metal sample (figure $\mathrm{S} 4 \mathrm{a}-\mathrm{b}$ ).

\section{Acknowledgements}

DS thanks Department of Science and Technology, Govt. of India for supporting a research project under Fast Track Scheme for Young Scientists (no. SR/FT/CS110/2010).

\section{References}

1. Khaled K F 2010 Mater. Chem. Phys. 124760
2. Popova A, Christov M, Raicheva S and Sokolova E 2004 Corros. Sci. 461333

3. Bentiss F and Lagrenée M 2010 J. Mater. Environ. Sci. 213

4. Quraishi M A and Sardar R 2003 J. Appl. Electrochem. Soc. 331163

5. Palomar-Pardavé M, Romero-Romo M, HerreraHernández H, Abreu-Quijano M A, Likhanova N V, Uruchurtu J and Juárez-García J M 2012 Corros. Sci. 54231

6. Ahamad I, Prasad R, Ebenso Eno E and Quraishi M A 2012 Int. J. Electrochem. Sci. 73436

7. Hassan N and Holze R 2009 J. Chem. Sci. 121693

8. Nataraja S E, Venkatesha T V, Tandon H C and Shylesha B S 2011 Corros. Sci. 534109

9. Khaled K F and Al-Qahtani M M 2009 Mater. Chem. Phys. 113150

10. Kovačević N and Kokalj A 2012 Mater. Chem. Phys. 137 331

11. Abd El-Maksoud S A and Fouda A S 2005 Mater. Chem. Phys. 9384

12. Obot I B, Obi-Egbedi N O and Umoren S A 2009 Int. J. Electrochem. Sci. 4863

13. El Ashry E S H, El Nemr A, Essawy S A and Ragab S 2008 Prog. Org. Coat. 6111

14. Popova A, Christov M and Vasilev A 2011 Corros. Sci. $\mathbf{5 3} 1770$

15. Abboud Y, Abourriche A, Saffaj T, Berrada M, Charrouf M, Bennamara A, Cherqaoui A and Takky D 2006 Appl. Surf. Sci. 2528178

16. Aljourani J, Golozar M A and Raeissi K 2010 Mater. Chem. Phys. 121320

17. Tang Y, Zhang F, Huc S, Cao Z, Wua Z and Jing W 2013 Corros. Sci. 74271

18. Chohan Z H, Shaibh A U, Rauf A and Supuran C T 2006 J. Enz. Inh. Med. Chem. 21741

19. Urra L, Gonzalez M P, Collado Isidro G and HernandezGalan R 2007 J. Mol Graphics Model 25680

20. Ozden S, Karates H, Yidiz S and Goker H 2004 Archiv der Pharmazie 337556

21. Nandi M M and Ray R 1987 Ind. J. Chem. 26A 345

22. Nandi M M and Ray R 1988 Ind. J. Chem. 27A 687

23. Ranjana and Nandi M M 2011 Ind. J. Chem. Tech. 1829

24. Frisch M J et al. 2009 Gaussian 09, Rev: D.01 (Gaussian, Inc., Wallingford, CT)

25. Pajkossy T 2005 Solid State Ionics 1761997

26. Rammelt U and Reinhard G 1987 Corros. Sci. 27373

27. Jorcin J B Orazem M E, Pébère N and Tribollet B 2006 Electrochim. Acta 511473

28. Obot I B and Obi-Egbedi N O 2010 Mater. Chem. Phys. 122325

29. Foo K Y and Hameed B 2010 Chem. Eng. J. 1562

30. ElBribri A, Tabyaoui M, Tabyaoui B, El Attari H and Bentiss F 2013 Mater. Chem. Phys. 141240

31. Fuchs-Godec R, Palavic M G and Tomic M V 2012 Ind. Eng. Chem. Res. 51274

32. Zarrouk A, Hammouti B, Dafali A and Bentiss F 2013 Ind. Eng. Chem. Res. 522560

33. Wang X, Wan Y, Zeng $\mathrm{Y}$ and Gu Y 2012 Int. J. Electrochem. Sci. 72403

34. Jørgensen C K 1963 In Inorganic complexes (London: Academic) p. 40

35. Bhattacharyya A 1989 Asian J. Chem. 2166 
36. Ghosh N N and Nandi M M 1976 Ind. J. Chem. 14A 778

37. Khaled K F 2010 Electrochim. Acta 556523

38. Gece G 2008 Corros. Sci. 502981

39. Panja S S and Chakraborty T 2003 J. Chem. Phys. 118 6200

40. Pauling L 1960 In The Nature of the Chemical Bond (New York: Cornell University Press)
41. Parr R G and Pearson R G 1983 J. Am. Chem. Soc. 105 7512

42. Geerlings P, De Proft F and Langenaeker W 2003 Chem. Rev. 1031793

43. Kabanda M M, Murulana L C, Ozcan M, Karadag F, Dehri I, Obot I B and Ebenso E E 2012 Int. J. Electrochem. Sci. 75035

44. Lukovits I, Kálmán E and Zucchi F 2001 Corrosion 573 